\title{
L'Oragé [2], Douna Loup (Paris, Mercure de France, 2015)
}

Jean-Joseph Rabearivelo et Esther Razanadrasoa, deux poètes sous l'occupation française

\section{Xavier Luce}

\section{OpenEdition}

\section{Journals}

Édition électronique

URL : https://journals.openedition.org/coma/607

DOI : $10.4000 /$ coma.607

ISSN : 2275-1742

\section{Éditeur}

Institut des textes \& manuscrits modernes (ITEM)

\section{Référence électronique}

Xavier Luce, «L'Oragé [2], Douna Loup (Paris, Mercure de France, 2015) », Continents manuscrits [En ligne], Comptes-rendus de parutions, mis en ligne le 20 octobre 2015, consulté le 20 janvier 2023. URL : http://journals.openedition.org/coma/607 ; DOI : https://doi.org/10.4000/coma.607 


\section{L'Oragé [2], Douna Loup (Paris, Mercure de France, 2015)}

Jean-Joseph Rabearivelo et Esther Razanadrasoa, deux poètes sous l'occupation française

\section{Xavier Luce}

\section{RÉFÉRENCE}

L'Oragé, Douna Loup, Paris, Mercure de France, 2015

Un roman saphique, songeai-je avec mépris. A mes yeux, influencée par Gilberto Freyre, je pensais qu'un roman devait conscientiser ses

lecteurs.

Maryse Condé, Mets et Merveilles, JC Lattès, 2015

\section{À partir d'un journal d'écrivain}

1 Sur quoi se fonde-t-elle, pour écrire ce roman, L'oragé ? Douna Loup aura puisé dans le journal intime de Jean-Joseph Rabearivelo ; si peu. Des cahiers qu'il ne brûla pas, ceux de 1933 jusqu'à son suicide en 1937 ; s'y trouvent deux allusions au personnage d'Esther Razanadrasoa, seulement.

Randriaminahy, en ce temps-là, attendait l'arrivée à Tananarive [...] du grand poëte et animatrice Esther Razanadrasoa, Anja-Z en littérature. Tous deux devaient se rencontrer quelques mois après, puis s'unirent pour de bon.

Je survins alors, qui avais été, moi aussi, en correspondance avec notre Sappho. Mais je n'ai pu arriver au bout de mes désirs que bien plus tard, après que la Lesbienne eut successivement vécu avec Raoely James, Malvoisin puis un poëtastre dont le nom m'échappe.

Calepins Bleus, le 8 avril $1935^{1}$ 
2 En ce temps-là remonte à une dizaine d'années, avant la mort de « notre Sappho ». Mais encore aujourd'hui (nous sommes alors en 1935) sa figure est dans tous les esprits, sur toutes les langues. Hier encore, ou était-ce samedi ? Rabe ne saurait le dire tant il est « sous le choc!", son nom était associé à celui de la défunte.

Pourquoi la grosse négresse de M. Dussol père m'a-t-elle [...] m'a-t-elle insinué que... Il paraît que c'est moi qui avais contribué à la mort de Razanadrasoa. Que cette admirable femme-poëte avait abusé de matières abortives pour se débarrasser de ma semence...

Et cela se dirait, en ville et cela, depuis la disparition de cette femme « complète »... Et me voici dévoré - non, certes, de remords - mais de doute, d'angoisse... C'est qu'en effet, il me souvient que j'ai bel et bien, et pas mal de fois, couché dans les bras de cette amie qui n'est plus...

Calepins Bleus, le 30 avril 1935

3 Une histoire de "coucherie ", des choses cachées... De resaka à L'oragé il y a toute l'épaisseur romanesque; un vide à combler. Affabulatrice, mentir-vrai ? Douna Loup écrit en anamorphose : d'une phrase des Calepins Bleus, elle tire plusieurs pages. Ainsi, l'arrivée d'Esther Razanadrasoa à Tananarive court des pages 35 à 38. Déjà, se creuse une conscience : Esther possède un regard rétrospectif, elle a connu Tananarive avant.

Quand je suis revenue dans ma Nanarivo j'ai vu toutes les strates. Je l'ai vue à nu, je l'ai vue sans eux. Car ils n'ont pas réussi leurs implants, ou si peu. Au fond nous sommes là, et même si nous sommes nés dans cette occupation de nos terres, nous sommes ce que nous sommes. Et ils sont en surface. Un jour la liberté sera déchirée.

Douna Loup, L'Oragé, p. 82

4 La vie des personnages se double d'un vécu lié au climat social : l'auteur intercale des évocations indépendantistes, des révoltes antérieures au récit, extérieures aux personnages. Rabearivelo n'a aucun lien avec les Menalamba du Nord, il n'est pas un révolutionnaire mais aspire à une décentralisation sur le modèle des Félibriges provinciaux. Il est régionaliste parce que le français coule dans ses veines. Douna Loup place Rabe à l'entrelacs d'événements et d'idées politiques; elles n'étaient pas les siennes mais elles étaient autour de lui : leur juxtaposition élabore une simultanéité romanesque. Esther Razanadrasoa est une force centripète, elle condense en elle un sentiment populaire et national qu'elle oppose à la fascination immodérée de son cadet pour l'Ailleurs.

Douna Loup a encerclé son sujet, sa découverte de Rabearivelo ne date pas d'hier pas plus que celle de la poésie malgache, de la langue même, cette «sauvage [...] malagasy qui traîne tard le soir dans la poussière des nonchalances ». Dans sa bibliothèque, La Coupe de Cendres, Presque-Songes ou Sari-Nofy, Traduit de la Nuit ou Tadika tamin'ny alina.. des recueils lorgnant entre Baudelaire et Verlaine; et puis, la romancière a vécu à Madagascar. La société dont il émane, l'île bienheureuse, entendre une Colonie bien gardée par son Gouverneur et sa milice, apparaît en filigrane. Des déclarations - « les droits de l'homme ne doivent pas être appris en malgache »- les évidences de l'époque. En 1910, alors en poste sur l'île, Jean Paulhan professait qu'il est en effet "stupide de leur enseigner que toute tyrannie est insupportable et que le seul bonheur est d'être un pays libre $»^{3}$. En éditant L'oragé, le Mercure de France fait un clin d'œil au directeur de la NRF et introducteur des hain-teny, Jean Paulhan défenseur de la culture malgache ; ironie des lettres. En tournant les pages du roman, "on peut, en 1866, lire... » les axiomes d'une civilisation à un moment donné de son expansion sans lesquelles on ne peut comprendre la colère, chez Esther, que suscite un article sur «le caractère rudimentaire de l'idiome local», un entrefilet à paraître, au nom de la liberté 
d'expression (sarcasme), dans les colonnes du Franco-Malgache où son ami Malvoiz occupe la première page.

\section{Un contexte social et historique}

6 Douna Loup offre à ses personnages une profondeur de champ, les met en prise avec les questions d'actualité. N'est-il pas préférable d'utiliser la main d'œuvre locale, moins onéreuse, plutôt que l'importer de métropole? Comment garantir la ligne de démarcation ?... Les coupures de presse agrémentent le récit oui mais ne l'écartèlent pas : Esther, Rabe et Malvoiz glissent entre les mots, se coulent dans des phrases bien tournées, malaxées. Madagascar? «Vue d'en haut, cosse de sauvagerie qui contient faune et flore, cosse magique à peine ouverte aux admirants ». Rabearivelo ? « Il avance dans le soir, il est cette langue vivante qui traverse la nuit»; oui, «il traverse l'agitation mais il n'est pas contaminé, il laisse sur lui le bruit couler et se concentre à son affaire "; il compte ses poèmes. Douna Loup, fascinée par Rabearivelo, habite son univers sensoriel et c'est par son prisme, seul, que s'envisage le roman.

Casimir l'enfant est une petite flûte.

Il faut attendre que ça souffle. Silence pour l'instant dans le coffre, mais on emmagasine de fines impressions. Il est un espace, une attente. Quelque chose vibre déjà au tout dedans. Les fleurs qui fument dans l'aurore, les premières voitures en ville et les opérations d'amour qu'il décrypte sur les visages.

Douna Loup, L'Oragé, p. 18

7 L'enfance de Rabe s'articule comme la justification rétrospective de son œuvre : déjà, il emmagasinait des sensations. Douna Loup postule les premières années du poète à partir de ses écrits ultérieurs. Non la reconstitution, de toute façon ne possédant pas les pièces nécessaires à une telle entreprise, la création d'un imaginaire. Douna Loup approche une vérité poétique, propose une immersion dans la galaxie Rabearivelo. Daniel Delas voudrait le contexte, une densité historique et sociale. A peine "effleuré ", seulement «en contrepoint »- les évocations et les citations de l'époque, brusques ouvertures sur la situation politique, confèrent un rythme et presque une menace sur le conte; une vie de bohème précaire- est-ce qu'un «lecteur ignorant des réalités malgaches $»^{4}$ sous l'occupation française se représentera le milieu que traversent les personnages?

8 L'édition des Calepins Bleus par l'équipe ITEM, quelques 1200 pages où se mêlent les digressions d'un indigène et dandy, offre un cadre de réflexion où accrocher les poèmes de Rabearivelo: de magnifiques sonnets pendant qu'on assène aux «agitateurs » des rappels à l'ordre, une bohème sous l'occupation. Un paradoxe que le huis-clos RabeEsther-Malvoiz laisse poindre ; il est en suspension, nimbe le récit. Fallait-il que L'oragé résolve cette apparente contradiction? Dilettantisme et colonialisme interfèrent. Rabe flirte avec une boursière des Beaux Arts de Paris : des toiles de Gauguin dans la tête, une bouteille à la main, « Pauline le tire chez elle [...]. C'est les beaux quartiers dit Rabe, [...] ils rient mais un peu plus loin [...] c'est un homme qui les arrête», un Sénégalais. Pas toujours natifs du Sénégal, ils appliquent les arrêtés du Gouverneur Général, en l'occurrence le couvre-feu indigène. Tout seul à l'angle d'une rue, sans qu'il ait besoin de la présence d'un Blanc, il s'interpose. Un système bien huilé mais une faille : Paulinequi-est-française au bras d'un Malgache. Pourquoi, "cette nuit», les laissera-t-il passer? Pauline demande le passage - « je dois emmener Rabe chez moi c'est urgent »elle l'obtient. Sa parole devient performative, c'est un ordre. D'où l'hébétude chez le 
"tirailleur " à sourire, "enfin quelque chose qui le sort du temps. Il sourit en les regardant dans la rue interdite", parce qu'elle vient contredire l'arrêté gouvernemental. La parole de Pauline frappe-t-elle de caducité la loi martiale? Il ne formule pas cette question, il « sort du temps».

Et le gardien ne veut pas sortir de son portique, il pleut fort. Ou peut-être qu'il s'en fout lui ce soir [...], il pense à la saison des pluies en Afrique de l'Ouest, il se demande comment sa mère se porte, et peut-être même qu'il est heureux cette nuit de les laisser passer dans l'interstice des réfractaires aux lois. Ou peut-être qu'il les poursuit, qu'il se fâche, qu'il se jette sur eux et les attrape comme des bêtes, qu'il déclenche l'alarme et qu'il les traine au poste. Mais le tirailleur sénégalais a été contaminé par cette Pauline qui vient d'ailleurs. Tout à coup il se sent d'ailleurs. Un homme venu de loin, tombé dans ce laps de noir et de rien.

Douna Loup, L'Oragé, p. 132

Cet «ailleurs" est le lieu de cette relation dont un membre est absent: ni colonial (fonctionnaire), colon (cultivateur) ou colonialiste (partisan). Cette absence offre un point de fuite à la situation. Ce glissement est permis par le choix du conte. Douna Loup recherche une conscience: à façonner un langage entre le malgache et le français, entre la presse coloniale et la poésie, à force de patience, elle se rapproche. Un cheminement, alana, confie-t-elle à son lecteur, dans «cette langue que j'utilise, ce français qui me sert à dire [...] pour l'heure pays étranger bloc lointain pour Rabe Casimir. Son pays, sa belle maternelle c'est ce lait ronono, ce rouge mena et ce ciel lanitra. / Je suis encore loin de lui ». Il faut aller le chercher.

Les personnages sont évanescents. Sûrement les émotions qu'exprime l'auteur cadrentelles avec les poèmes de Rabearivelo. Mais où la diglossie, où comprend-on que cela se passe sous un régime totalitaire s'enquiert Daniel Delas? Le cadre spatio-temporel entoure les poètes, à combattre la suprématie d'une langue, tout un peuple, sur l'autre, Anja-Z s'y évertue, à n'écrire qu'en sa «malagasy » quand Rabe harmonise, "veut croire aux complémentarités et à l'ouverture » $:$ se " mallarmiser ${ }^{5}$, l'ambition, l'utopie qui le révolte contre les petitesses de certains, certain toujours qu'une relation peut se nouer, une civilisation se parfaire. En somme, qu'un contact est possible.

Mais c'est la base qui est biaisée, Rabe, le rapport de supériorité. moi c'est ce qui me renverse aux Beaux-Arts, on me demande de former des étudiants à notre façon, de les prendre de haut comme si c'était des gosses, mais on aurait à apprendre de vous, à échanger à savoir ce qu'elles sont vos expressions plastiques. Vous n'avez pas peint et alors, vous avez fait d'autres choses, non?

Douna Loup, L'Oragé, p. 131

\section{Identité, identification}

11 Rabe est le postulat d'un système de beaucoup supérieur au métissage. Homme de cultures, bilingue oui tant qu'on voudra admettre que cette capacité l'empêche de se définir autrement que par rapport au vazaha ${ }^{6}$ et selon une ascendance royale. A ses amis de France, il fait valoir qu'il est un roi déchu non un fils d'esclave : en effet, « la famille est de lignée noble, des enfants de Zanadralambo, mais leur fortune sur l'esclavage s'est effondrée avec l'abolition, avec la colonisation ne reste que le souvenir des richesses, du vent qui chante ancestral, d'un nom qui coule aristocrate ${ }^{7}$. Rabearivelo cherche la reconnaissance de son identité plutôt que de son altérité. La réception du prince Aniaba à la cour de Louis XIV incarne cette figure nobiliaire que consacrent ces paroles du roi Soleil : «il n'y a donc pas plus de différence entre vous et 
moi que du noir au blanc » adressa-t-il au digne Aniaba à son retour en Afrique pour les funérailles de son père. Yambo Ouologuem cerne bien l'enjeu de la rencontre en recopiant ou réécrivant? ces lignes de Robert Delavignette.

Quand Louis XIV disait à Aniaba : «il n'y a donc pas plus de différence entrez vous et moi que du noir au blanc ", il marquait que tous deux étaient [...] solidaires dans la royauté, et qu'ils pouvaient être différents par la couleur tout en étant unis par l'identité de leur nature royale. Et maintenant, par extension, c'est de l'identité d'une nature royale qu'il s'agit entre l'Afrique et nous.

Yambo Ouloguem, Le Devoir de violence, Paris, Le Serpent à Plumes, 2003, p. 65

12 L'identification de Rabearivelo à la monarchie française n'a rien de si incongru : celle-ci se lit comme une anamorphose de celle-là. Une des raisons pour laquelle son œuvre est à l'abri de toute violence coloniale - on sait que la politique étouffe l'art, qu'un écrivain trop politique vieillit mal - est que Rabearivelo ne l'envisage jamais. Ou si peu, Esther lui raconte.

Ils nous ont aussi enfermés, Rabe. Ils ont fusillé, il faut dire. il y a des ouvertures terreurs. Tu connais les Menalamba? Nos révoltés du Nord, nos chefs de guérilla? Tu connais Rabezavana?

Oui.

Oui.

Ils passent à autre chose

Douna Loup, L'Oragé, p. 83

13 Il évacue, on sait tout ça. Ou bien, Douna Loup le suggère, il croit à l'osmose, que la Beauté, dans son panthéon, réunit les «H. C. "), les hommes et les femmes " complètes" comme Esther. Car ici, le sang se moque de la Couleur", les Lettres unissent sur un pied de supériorité indigène et citoyen. Pour avoir l'ambition de rêver "le miracle d'une prose poétique, musicale sans rythme et sans rime, assez souple et assez heurtée pour s'adapter aux mouvements lyriques de l'âme, aux ondulations de la rêverie, aux soubresauts de la conscience ${ }^{10}$ ». Rabearivelo devient français parce qu'il l'écrit ; mieux, le sublime. Dans ces jours d'ambition, il rêvait oui, qu'un de ses pairs l'adoube, s'entendre dire qu'« il manie la langue française comme aucun blanc ne saurait le faire ${ }^{11}$ ", cela qu'André Breton, dans un excès de franchise, adressera à Aimé Césaire une dizaine d'années après son suicide et une guerre mondiale. En lui reconnaissant un style, on cesserait de parler de ses incorrections ou de son impersonnalité. Sur ce piédestal, il resterait malgache parce qu'il aurait pu être français, s'il avait voulu, car on lui a maintes fois proposé assure-t-il dans le Colonial \& Malagasy de son ami Malvoiz. Noblesse de ne pas désirer ce que l'on mérite plus qu'aucun autre, signe d'une plus haute aristocratie: quand une fange urbaine et européanisée brigue une naturalisation comme un hobereau s'achète une particule, lui, aspire à "des bonheurs supérieurs aux leurs, plus vastes et plus raffinés $»^{12}$ sans doute. C'est dans les lettres qu'il se réalise, dans sa fréquentation «bestiale » de la littérature étrangère. Il hante sa ville et débouche sur Paris, la rue de Rome, la butte Montmartre... Esther l'incite à un autre regard, à se concentrer sur lui-même et cette « Nanarivo ».

Je suis née dix ans avant toi, Rabe, mais je n'ai de souvenir que sous la Colonie. Et finalement c'est étrange, je me dis souvent que ce n'est rien cette sur-couche... cette présence banale des colons dans la ville. J'ai grandi avec, en me demandant audedans quelles étaient leurs places ici. J'ai voyagé ensuite, ailleurs, j'ai vu les visages de nos villages sans vazaha, sans bureau. Ça m'a aidée je crois. Aidée de nous voir nous, nous seuls.

Douna Loup, L'Oragé, p. 82 

de l'Histoire, à ce qu'il semble n'avoir pas vu ou pas pris en compte parce qu'il envisageait les choses d'un point de vu Merina plutôt que malgache; il se définit essentiellement comme un Grand Hova à côté du Français. Esther parle d'un nousmêmes, premier ferment d'une conscience nationale, et d'un sans eux. Et vraiment, "Rabearivelo est une langue ", mais qu'est-ce qu'une langue sans locuteur, sans corps? Un idéal qui «traverse la nuit » et l'on dira la langue de Rabearivelo comme on le dit de Molière, c'est la phrase d'ouverture de l'émission bilingue malgache Écho du Capricorne sur radio Fréquence Paris Plurielle ${ }^{13}$. Le poète oragé est ce mouvement féroce d'une culture vers une autre dans l'envie de l'explorer, casser, mamaky, les frontières culturelles pour les ouvrir les unes sur les autres, les appuyer l'une contre l'autre, manankina ${ }^{14}$.

\section{BIBLIOGRAPHIE}

Jean-Joseph Rabearivelo, « Calepins Bleus », in CEuvres complètes, t. I, édition critique coordonnée par Serge Meitinger, Liliane Ramarosoa et Claire Riffard, Paris, CNRS Éditions, coll. « Planète Libre ", 2010.

Jean-Joseph Rabearivelo, « Presque-Songes / Sari-Nofy », in Cuvres complètes, t. II, édition critique coordonnée par Serge Meitinger, laurence Ink, Liliane Ramarosoa et Claire Riffard, Paris, CNRS Éditions, coll. « Planète Libre », 2012.

Yambo Ouloguem, Le Devoir de violence, Paris, Le Serpent à Plumes, 2003.

Charles Baudelaire, « À Arsène Housaye », Petits poëmes en prose (Le Spleen de Paris), édition de 1869.

Jean Paulhan et Madagascar (1908-1910), introduction de Roger Judrin, présenté par Jacqueline Frédéric Paulhan, collection « Cahiers Jean Paulhan » ( ${ }^{\circ}$ 2), Paris, Gallimard, 1982.

André Breton, « Un grand poète noir », Martinique, charmeuse de serpents repris dans Aimé Césaire, Cahier d'un retour au pays natal, Présence africaine.

Le Journal de Madagascar franco-malgache, titre courant Journal [Le] franco-malgache puis Francomalgache, paraissant les mardi et vendredi, bilingue, gérant P. Étienne puis G. Dussol, 2 févr. 1923 - 29 mai 1925.

Le Colonial et le Malagasy (réunis), Colonial \& Malagasy, paraissant le dimanche, Tananarive, 10 janvier 1932, puis devient le Malagasy, Organe de défense des intérêts malgaches, gérant Ch. Ranaivo.

\section{NOTES}

1. Jean-Joseph Rabearivelo, "Calepins Bleus", in Euvres complètes, t.I, édition critique coordonnée par Serge Meitinger, Liliane Ramarosoa et Claire Riffard, Paris, CNRS Editions, coll. Planète Libre, 2010. 
2. Rabearivelo, dans ses Calepins Bleus, emprunte au malgache le terme pour désigner la rumeur de la ville, les ragots, les on-dits.

3. Dans une lettre à ses proches reproduite dans Jean Paulhan et Madagascar, (1908-1910), Paris, NRF-Gallimard, p. 77-78.

4. $\mathrm{Cf}$ article de Daniel Delas

5. Rabearivelo, dans ses Calepins Bleus, le 6 mai 1933, forme le néologisme pour exprimer sa vision de la langue malgache en face du français.

6. Désigne l'étranger, ici l'Européen et particulièrement l'occupant.

7. Douna Loup, L'oragé, Mercure de France, 2015, p. 83, p. 17-18

8. Acronyme que Rabearivelo emploie dans ses Calepins Bleus pour désigner les gens de la plus haute aristocratie, les lettrés, les parfaits humanistes (Cf. ce qu'il écrit le 15 avril 1936 au sujet de la «Pléiade lyonnaise »: à rapprocher de l'adjectif féminisée par Douna Loup à l'endroit d'Esther).

9. C'est dans les Calepins Bleus, en ces termes, qu'il justifie son identification au nationalisme et à la monarchie française ; le 15 janvier 1934 il justifie son « adhésion morale [...]. Purement morale. » à Charles Maurras et Léon Daudet « au nom de l'Esprit ».

10. Baudelaire, «À Arsène Houssaye ", Petits poëmes en prose (Le Spleen de Paris), édition de 1869, p. 2

11. André Breton, "Un grand poète noir ", Martinique, charmeuse de serpents repris dans Aimé Césaire, Cahier d'un retour au pays natal, Présence africaine

12. Baudelaire, « XII. Les Foules », op. cit. p. 32

13. Une émission hebdomadaire de $20 \mathrm{~h} 30$ à $22 \mathrm{~h}$ sur Radio FPP (106.3 ou rfpp. net)

14. L'oragé, op. cit., p. 114-115

\section{AUTEURS}

\section{XAVIER LUCE}

Etudiant en Master II, Sorbonne 